\title{
Successful treatment of oral verrucous hyperplasia with topical 5-aminolevulinic acid-mediated photodynamic therapy
}

\author{
Hsin-Ming Chen ${ }^{a, b}$, Chin-Tin Chen ${ }^{c, d}$, Hsiang Yang ${ }^{b}$, Mark Yen-Ping Kuo ${ }^{a, b}$, \\ Ying-Shiung Kuo ${ }^{a, b}$, Wan-Hong Lan ${ }^{a, b}$, Yi-Ping Wang ${ }^{a, b}$, \\ Tsuimin Tsai ${ }^{\mathrm{e}, *}$, Chun-Pin Chiang ${ }^{\mathrm{a}, \mathrm{b}, *}$
}

\author{
a Department of Dentistry, National Taiwan University Hospital, National Taiwan University, \\ 1, Chang-Te Street, Taipei 100, Taiwan \\ ${ }^{\mathrm{b} S}$ School of Dentistry, National Taiwan University, 1, Chang-Te Street, Taipei 100, Taiwan \\ 'Graduate Institute of Oral Biology, National Taiwan University, 1, Chang-Te Street, Taipei 100, Taiwan \\ ${ }^{\mathrm{d}}$ Center for Optoelectronic Biomedicine, College of Medicine, National Taiwan University, \\ 1, Chang-Te Street, Taipei 100, Taiwan \\ ${ }^{\mathrm{e}}$ Graduate Institute of Biomedical Materials, Taipei Medical University, 250, Wu-Hsing Street, \\ Taipei 110, Taiwan
}

Received 6 November 2003; accepted 10 December 2003

\section{KEYWORDS \\ 5-aminolevulinic acid; \\ Oral verrucous \\ hyperplasia; \\ Photodynamic therapy}

\begin{abstract}
Summary Previous studies have shown a selective accumulation of 5-aminolevulinic acid (ALA)-derived protoporphrin IX (PpIX) in oral premalignant and malignant tissues. This provides a biologic rationale for the clinical use of ALA-mediated PDT (ALA-PDT) for oral premalignant and malignant lesions. In this study, five patients with oral verrucous hyperplasia $(\mathrm{OVH})$ were treated with a new protocol of ALA-PDT composed of multiple 3-min irradiations with a light emitting diode (LED) red light at $635 \pm 5 \mathrm{~nm}$ separated with several $3-\mathrm{min}$ rests for a total of $1000 \mathrm{~s}$ (fluence rate, $100 \mathrm{~mW} / \mathrm{cm}^{2}$; light exposure dose, $100 \mathrm{~J} / \mathrm{cm}^{2}$ ) after topical application of $20 \%$ ALA for 1.5 or $2 \mathrm{~h}$. Topical ALA-PDT was repeated once a week until the complete regression of the lesion. Complete regression of all OVH lesions was observed after 1-3 treatments (average, 2 treatments) of topical ALA-PDT. At an average follow-up of 5.6 months (range, 3-11 months), all the five OVH patients were free of tumor recurrence. We conclude that topical ALA-PDT with fractionated irradiations by an LED red light at $635 \pm 5 \mathrm{~nm}$ is an effective and successful treatment modality for $\mathrm{OVH}$.

(c) 2004 Elsevier Ltd. All rights reserved.
\end{abstract}

"Corresponding authors. Tel.: +886-2-2312-3456x6855; fax: +886-2-2389-3853 (C.-P. Chiang), tel./fax: +886-2-2739-0082 (T. Tsai).

E-mail addresses: tmtsai00@tmu.edu.tw (T. Tsai), cpchiang@ha.mc.ntu.edu.tw (C.-P. Chiang).

\section{Introduction}

Oral verrucous hyperplasia $(\mathrm{OVH})$ is a premalignant lesion that may transform into a verrucous 
carcinoma or a squamous cell carcinoma (SCC). Traditional treatment for $\mathrm{OVH}$ is total surgical excision that always leads to scar formation for a large OVH lesion. Photodynamic therapy (PDT) is another effective treatment option for human premalignant and malignant lesions that heal with remarkably little scar. ${ }^{1}$ PDT involves two individually non-toxic components, light and photosensitizer, that work together to induce cellular and tissue destruction in an oxygen-dependent manner. This technique is based on the administration of an exogenous photosensitizer to render tumor tissue sensitive to light of a specific wavelength. The photosensitizers are normally inert and have a selective affinity to tumor tissues. When a photosensitizer in tissues is activated by a light of specific wavelength, it transfers energy from light to molecular oxygen, resulting in generation of reactive oxygen species (ROS). ${ }^{1}$ There are three main mechanisms by which PDT mediates tumor destruction. Firstly, the ROS can kill tumor cells directly. Secondly, PDT can damage the tumorassociated vasculature, leading to thrombus formation and subsequent tumor infarction. Thirdly, PDT can also activate an immune response against tumor cells. ${ }^{1}$

5-Aminolevulinic acid (ALA) has been successfully used in the diagnosis and treatment of neoplastic tissues. ${ }^{2-19}$ ALA itself is not a photosensitizer but serves as the biological precursor of the photosensitizer, protoporphyrin IX (PpIX), in the heme biosynthesis pathway. There are two rate-limiting steps in this pathway. One is the first step of forming ALA from glycine and succinyl CoA, which is regulated by heme via a negative feedback mechanism. The other ratelimiting step is the conversion of PpIX to heme, and this process is controlled by ferrochelatase, which adds a ferrous iron to PpIX to form the heme. Exogenous ALA administration short-circuits the first step of porphyrin synthesis and subsequently leads to the accumulation of PpIX in the tissue. Furthermore, PpIX accumulation could be the result of a decreased conversion of PpIX to heme in tumor cells as a result of a decreased ferrochelatase activity. ${ }^{2,3}$

Cutaneous photosensitivity is a common adverse effect in clinical practice of PDT. Compared to Photofrin ${ }^{\circledR}$-mediated PDT, ALA-PDT has been shown to have less cutaneous photosensitivity. 2,3 ALA can be rapidly cleared from the tissues and the body within $48 \mathrm{~h}$. Thus, patients after ALA-PDT treatment have no problem of prolonged skin photosensitivity.

PDT with systemically or topically administered ALA (systemic or topical ALA-PDT) has been used for treatment of human premalignant and malignant lesions from the oral cavity, ${ }^{4-7}$ esophagus, ${ }^{8-10}$ uterine cervix, ${ }^{11}$ urinary bladder, ${ }^{12,13}$ and skin $^{14,15}$ with relatively good clinical outcomes. ALA-PDT at least has the following advantages over conventional treatments: it is non-invasive; it produces excellent cosmetic results; it is well tolerated by patients; it can be applied to patients who refuse surgery or have pacemakers and bleeding tendency; and it can be applied repeatedly without cumulative toxicity. ${ }^{16}$ Previous studies by Leunig and his colleagues ${ }^{17-19}$ demonstrated that topically applied ALA can be selective for oral premalignant and malignant tissues. In addition, ALA-induced PpIX has good fluorescent properties; therefore, the level of PpIX in tissues can be monitored by fluorescence spectroscopy and PDT can be carried out at the time point when the PpIX reaches its maximal concentration in tissues.

In this study, we tested the efficacy of a new treatment protocol of PDT using a topical application of $20 \%$ ALA onto oral lesions followed by multiple 3-min fractionated irradiations with a light emitting diode (LED) red light at $635 \pm 5 \mathrm{~nm}$ for five patients with $\mathrm{OVH}$. OVH lesions rather than other premalignant lesions were selected because $\mathrm{OVH}$ lesions are less hyperkeratotic than oral leukoplakia; this may lead to easy diffusion of topically applied ALA into lesional epithelial cells. Furthermore, $\mathrm{OVH}$ lesions present as single or multiple exophytic or elevated masses with welldemarcated border; it is easy to observe the changes in size of the lesion after PDT. In addition, we chose topical application but not systemic administration to deliver ALA into OVH lesions, because systemic administration of ALA to the patients has the side effects of nausea, vomiting and elevation of blood levels of bilirubin and liver enzymes (e.g. aspartate transaminase, AST). ${ }^{5}$ On the contrary, direct local application of ALA onto oral lesions does not have any systemic side effects. ${ }^{17-19}$ In this study, an LED light device was used because it was a simpler, smaller, lighter, cheaper, and more portable light source than the laser machine. Moreover, it deserved to test because it may have the high potential to replace the laser machine in clinical use for PDT in the near future.

\section{Material and methods}

\section{Patients}

Five male patients (aged 37-64 years, mean age $52 \pm 11$ years) with $\mathrm{OVH}$ were recruited from the 
Table 1 Clinicopathological data as well as topical ALA-PDT and its clinical outcome for five patients with oral verrucous hyperplasia (OVH)

\begin{tabular}{|c|c|c|c|c|c|c|c|c|c|}
\hline $\begin{array}{l}\text { Patient } \\
\text { no. }\end{array}$ & Age & Sex & $\begin{array}{l}\text { Lesion } \\
\text { location }\end{array}$ & $\begin{array}{l}\text { Patholog- } \\
\text { ical } \\
\text { diagnosis }\end{array}$ & $\begin{array}{l}20 \% \text { ALA } \\
\text { used per } \\
\text { treatment }\end{array}$ & $\begin{array}{l}\text { Incubation } \\
\text { period from } \\
\text { ALA applica- } \\
\text { tion to light } \\
\text { treatment }\end{array}$ & $\begin{array}{l}\text { Number } \\
\text { of ALA- } \\
\text { PDT } \\
\text { used }\end{array}$ & $\begin{array}{l}\text { Clinical } \\
\text { outcome } \\
\text { of } \\
\text { ALA-PDT }\end{array}$ & Follow-up \\
\hline 1 & 44 & Male & $\begin{array}{l}\text { Left mouth } \\
\text { angle }\end{array}$ & $\mathrm{OVH}$ & $0.3 \mathrm{cc}$ & $1.5 \mathrm{~h}$ & 2 & $\begin{array}{l}\text { Complete } \\
\text { response }\end{array}$ & $\begin{array}{l}6.5 \text { months, } \\
\text { no recur- } \\
\text { rence }\end{array}$ \\
\hline 2 & 64 & Male & $\begin{array}{l}\text { Right mouth } \\
\text { angle }\end{array}$ & $\begin{array}{l}\text { Lesion } \\
\text { too small, } \\
\text { biopsy } \\
\text { not done }\end{array}$ & $0.1 \mathrm{cc}$ & $1.5 \mathrm{~h}$ & 1 & $\begin{array}{l}\text { Complete } \\
\text { response }\end{array}$ & $\begin{array}{l}11 \text { months, } \\
\text { no recur- } \\
\text { rence }\end{array}$ \\
\hline 3 & 56 & Male & $\begin{array}{l}\text { Left buccal } \\
\text { mucosa }\end{array}$ & $\mathrm{OVH}$ & $0.6 \mathrm{cc}$ & $2 \mathrm{~h}$ & 2 & $\begin{array}{l}\text { Complete } \\
\text { response }\end{array}$ & $\begin{array}{l}3 \text { months, no } \\
\text { recurrence }\end{array}$ \\
\hline 4 & 37 & Male & $\begin{array}{l}\text { Right lower } \\
\text { labial mucosa }\end{array}$ & $\mathrm{OVH}$ & $0.3 \mathrm{cc}$ & $1.5 \mathrm{~h}$ & 3 & $\begin{array}{l}\text { Complete } \\
\text { response }\end{array}$ & $\begin{array}{l}3.5 \text { months, } \\
\text { no recur- } \\
\text { rence }\end{array}$ \\
\hline 5 & 57 & Male & $\begin{array}{l}\text { Right lower } \\
\text { labial mucosa }\end{array}$ & $\mathrm{OVH}$ & $0.3 \mathrm{cc}$ & $1.5 \mathrm{~h}$ & 2 & $\begin{array}{l}\text { Complete } \\
\text { response }\end{array}$ & $\begin{array}{l}4 \text { months, no } \\
\text { recurrence }\end{array}$ \\
\hline
\end{tabular}

Department of Oral and Maxillofacial Surgery, National Taiwan University Hospital (NTUH) from 2002 to 2003 (Table 1). Clinical diagnosis of $\mathrm{OVH}$ was made when patients showed characteristic single or multiple elevated verrucous lesions without marginal induration on the oral mucosa. Clinical diagnosis was confirmed by histopathological examination of the biopsy specimens taken from the characteristic part of the $\mathrm{OVH}$ lesion at the patient's first visit. The histological criteria for a diagnosis of OVH were: (1) epithelial hyperplasia with parakeratosis or hyperkeratosis and verrucous surface, and (2) no invasion of the hyperplastic epithelium into the lamina propria as compared to adjacent normal mucosal epithelium. The OVH lesions were located at the mouth angle in two patients, at the buccal mucosa in one patient, and at the labial mucosa in two patients (Table 1). All the five $\mathrm{OVH}$ patients were both $\mathrm{AQ}$ chewers and smokers. Informed consent was obtained from each patient before biopsy procedure and before PDT. This study was reviewed and approved by the Human Investigation Review Committee at the National Taiwan University Hospital.

\section{ALA preparation}

The formulation of ALA contained 25\% Pluronic F127 and 1\% Carbopol 971P. Pluronic F127 was obtained from BASF (Mount Olive, NJ, USA). Carbopol 971P was a gift from BF Goodrich. Double concentrated gels of Pluronic F127 and Carbopol 971P in distilled water were separately prepared prior to mixing. Prior to use, $200 \mathrm{mg}$ of ALA was mixed with $800 \mathrm{mg}$ of the gel and packed into a needleless 1 -cc syringe. The formulation was used within $3 \mathrm{~h}$ after the preparation.

\section{Fluorescence spectroscopy}

ALA diffusing into cells is metabolized into PpIX in the mitochondrial matrix and cytosol. To monitor the conversion of ALA into PpIX and to determine when the PpIX reaches its peak level in the lesional epithelial cells, ALA-induced PpIX fluorescence spectra at 410-nm excitation were measured $1,1.5,2$, and $2.5 \mathrm{~h}$ after local application of $20 \%$ ALA to each oral lesion. This fluorescence spectroscopy was performed by using a handheld optical fiber probe attached to a spectrofluorometer (SkinSkan, JC Inc., Urbana, IL, USA) at the patient's second visit. A monochromator with a $150 \mathrm{~W}$ ozone-free Xenon lamp provided the excitation light. The excitation light was guided to illuminate samples by one arm of a Y-type quartz fiber bundle, and the emission fluorescence was collected by another arm of the fiber bundle. The emission fluorescence spectra were collected from 450 to $750 \mathrm{~nm}$ in 3-nm increments during the measurement. A personal computer was used to analyze the fluorescence spectral data of each $\mathrm{OVH}$ lesion in order to find out the time point at which the PpIX reaches its peak level in the lesional epithelial cells. This time point was the most appropriate occasion for each particular $\mathrm{OVH}$ lesion to deliver the light treatment. 


\section{PDT}

PDT was performed once a week starting from the patient's third appointment. At the day of treatment, 20\% ALA was applied to each OVH lesion upon patient's arrival. To avoid the dilution of the ALA by the saliva, three cotton rolls were placed onto the orifices of bilateral Stensen's and Wharton's ducts before ALA application. In addition, the patient was asked to lie on the dental chair with the mouth being opened and the doctor performing the treatment controlled the leaked saliva by intermittent suctions during the initial 10 min after topical application of ALA. The light treatment composed of multiple 3-min irradiations with an LED red light at $635 \pm 5 \mathrm{~nm}$ separated with several 3 -min rests for a total of $1000 \mathrm{~s}$ (fluence rate, 100 $\mathrm{mW} / \mathrm{cm}^{2}$; light exposure dose, $100 \mathrm{~J} / \mathrm{cm}^{2}$ ) was given 1.5 or $2 \mathrm{~h}$ after topical ALA application. If possible, the whole lesion was irradiated using one spot of light. If the lesion was too large to be covered by one spot of light or separated into multiple portions, several spots of light were applied until the whole lesion was completely illuminated. Light treatments were carried out under local anesthesia using $2 \%$ lidocaine with the patient in clear consciousness. The doctors performing the treatment held the LED light device during the whole treatment period. The tip of the LED light device was kept as close to the surface of $\mathrm{OVH}$ lesion as possible. Analgesics were prescribed to the patients to control the post-PDT pain. Repeated PDT was performed once a week until the complete regression of the lesion. The patients were then followed up once a week in the first month and once a month thereafter. In addition, clinical photographs were taken at each patient's visit to evaluate the outcome of PDT. Lesion response was characterized as follows: complete response, lack of detectable lesion confirmed by clinical evaluation; partial response, reduction of lesion by at least $20 \%$ in diameter; and no response, reduction of lesion by less than $20 \%$ in diameter.

\section{Results}

Fluorescence emission spectra of the OVH lesions were collected after topical ALA application. As shown in Fig. 1, at 410-nm excitation the spectra consisted of two emission peaks: one at $470 \mathrm{~nm}$ and the other at $635 \mathrm{~nm}$. The 470-nm emission peak was attributed to the autofluorescence from the $\mathrm{OVH}$ lesion, as the fluorescence intensity did not change during the whole study period. The $635-\mathrm{nm}$ emission peak was attributed to PpIX fluorescence from the $\mathrm{OVH}$ lesion; the PpIX accumulation arrived at a significant level at $1 \mathrm{~h}$ and reached a maximum level at 1.5 or $2 \mathrm{~h}$ after topical application of ALA.

In this study, biopsy of the OVH lesion was performed for four patients. All the four biopsy specimens showed verrucous hyperplasia (Table 1). The histological section of an OVH specimen taken from patient 1 (Fig. 2A) is shown in Fig. 2B. Biopsy was not done for patient 2, because the lesion was too small (Fig. 2E). This lesion was diagnosed as an $\mathrm{OVH}$

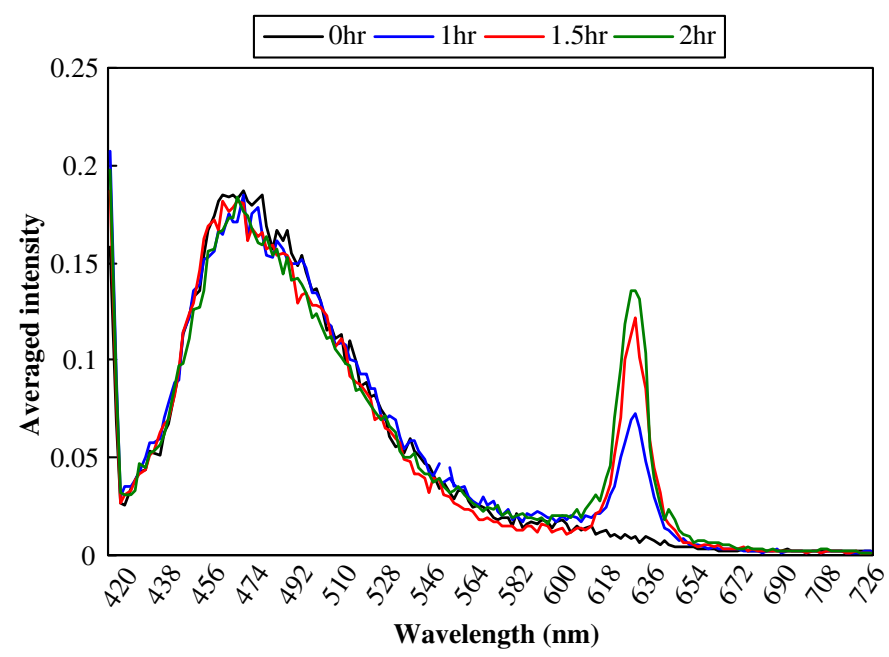

Figure 1 Normalized fluorescence emission spectra at 410-nm excitation obtained in situ at different time points after topical application of ALA onto oral verrucous hyperplasia $(\mathrm{OVH})$ lesion. The emission peak at $470 \mathrm{~nm}$ is attributed to autofluorescence from the $\mathrm{OVH}$ lesion and that at $635 \mathrm{~nm}$ is attributed to ALA-induced protoporphyrine IX from the $\mathrm{OVH}$ lesion. Fluorescence intensities are reported in calibrated units. 

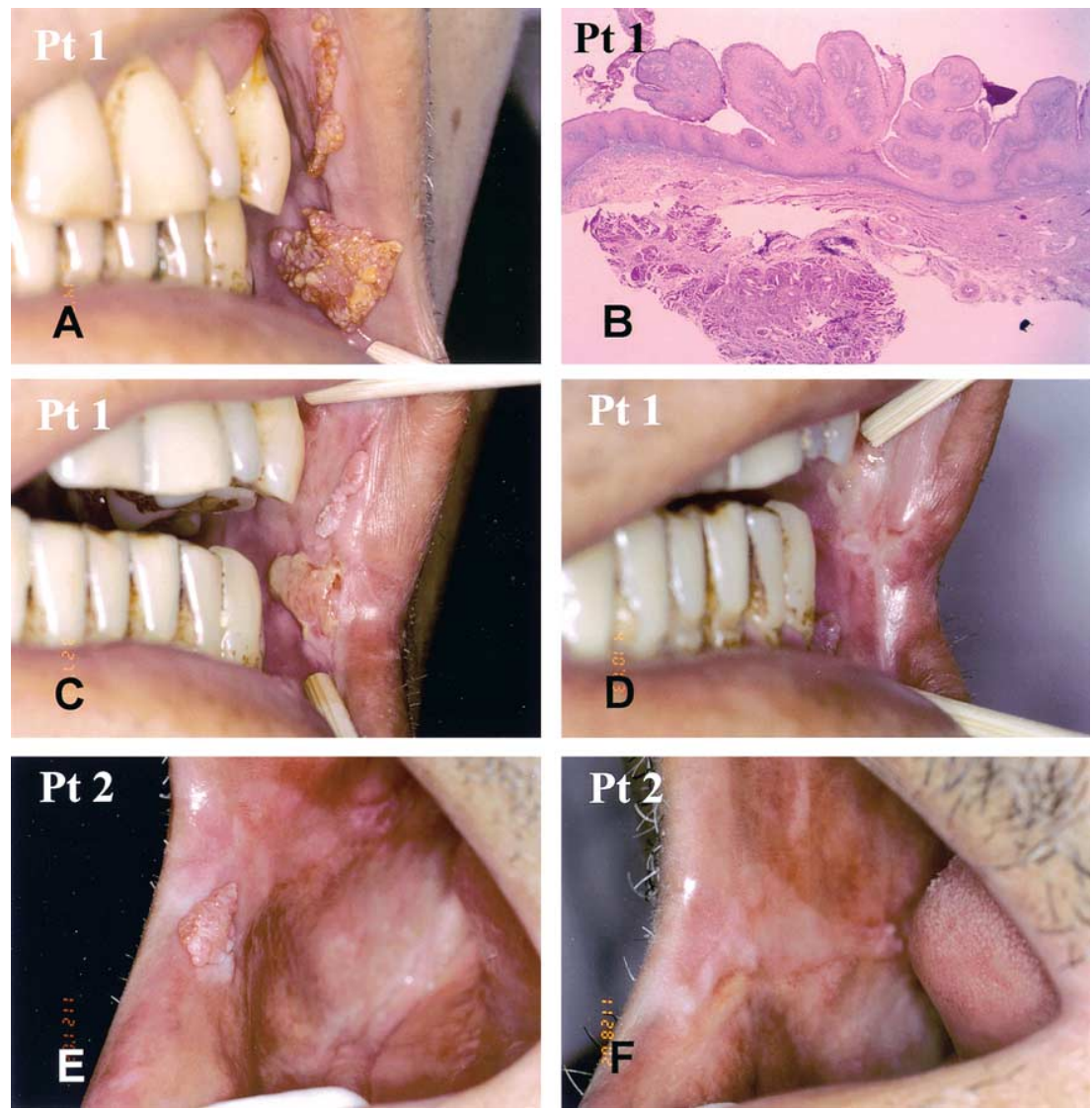

Figure 2 Clinical and histologic photographs of patient 1 and patient 2 with oral verrucous hyperplasia (OVH). (A) Two OVH lesions at the left mouth angle of patient 1 before biopsy. (B) Biopsy of the lower lesion in Fig. 1A showing characteristic feature of verrucous hyperplasia (hematoxylin and eosin stain, $\times 10$ ). (C) Clinical picture of patient 1 before PDT. (D) Clinical picture of patient 1 after 2 treatments of ALA-PDT showing complete regression of the OVH lesion. (E) An OVH lesion at the right mouth angle of patient 2 before PDT. (F) Clinical picture of patient 2 after one treatment of ALA-PDT showing complete regression of the OVH lesion.

lesion based on the characteristic clinical appearance only.

All OVH lesions were treated with topical ALAPDT once a week. At the day of the treatment, the lesions were topically applied with $0.1-0.6 \mathrm{cc}$ (average, $0.32 \mathrm{cc}$ ) of the $20 \%$ ALA solution, depending on the size of the lesions. Because fluorescence spectroscopy performed at each patient's second visit showed that PpIX reached its maximal level in the $\mathrm{OVH}$ lesion at 1.5 or $2 \mathrm{~h}$ after topical application of ALA, each OVH lesion was treated with multiple 3-min fractionated irradiations with an LED red light at $635 \pm 5 \mathrm{~nm}$ for a total of $1000 \mathrm{~s}$ (fluence rate, $100 \mathrm{~mW} / \mathrm{cm}^{2}$; light exposure dose, $100 \mathrm{~J} / \mathrm{cm}^{2}$ ) after 1.5 or $2 \mathrm{~h}$ of incubation period (Table 1). An average of 2 treatments (range, 1-3 treatments) of ALA-PDT were needed to achieve the complete regression of the lesions (Table 1, Fig. 2C-F, and Fig. $3 \mathrm{~A}-\mathrm{F}$ ). In addition, no recurrence was found after a follow-up period of 3-11 months (mean, 5.6 months) (Table 1).

\section{Discussion}

In this study, five $\mathrm{OVH}$ patients were treated with a new topical ALA-PDT protocol composed of multiple 3-min fractionated irradiations with an LED red light at $635 \pm 5 \mathrm{~nm}$ for a total of $1000 \mathrm{~s}$. Complete regression of the lesions was found after 1-3 treatments in every case, indicating a successful and effective treatment with this new PDT protocol. There were four characteristic features of this study. First, we first tested the efficacy of a topical ALA-PDT on a specific group of oral premalignant lesion- $\mathrm{OVH}$. Second, a new formulation of topical ALA preparation was used for the treatment. Third, an LED red light device was used for irradiation of $\mathrm{OVH}$ lesions. Fourth, a new PDT protocol of multiple 3-min fractionated irradiations was tested in this study. Although a randomized, double-blind, placebo-controlled trial was needed to verify this new treatment modality, we suggest that the successful clinical outcome of our topical 

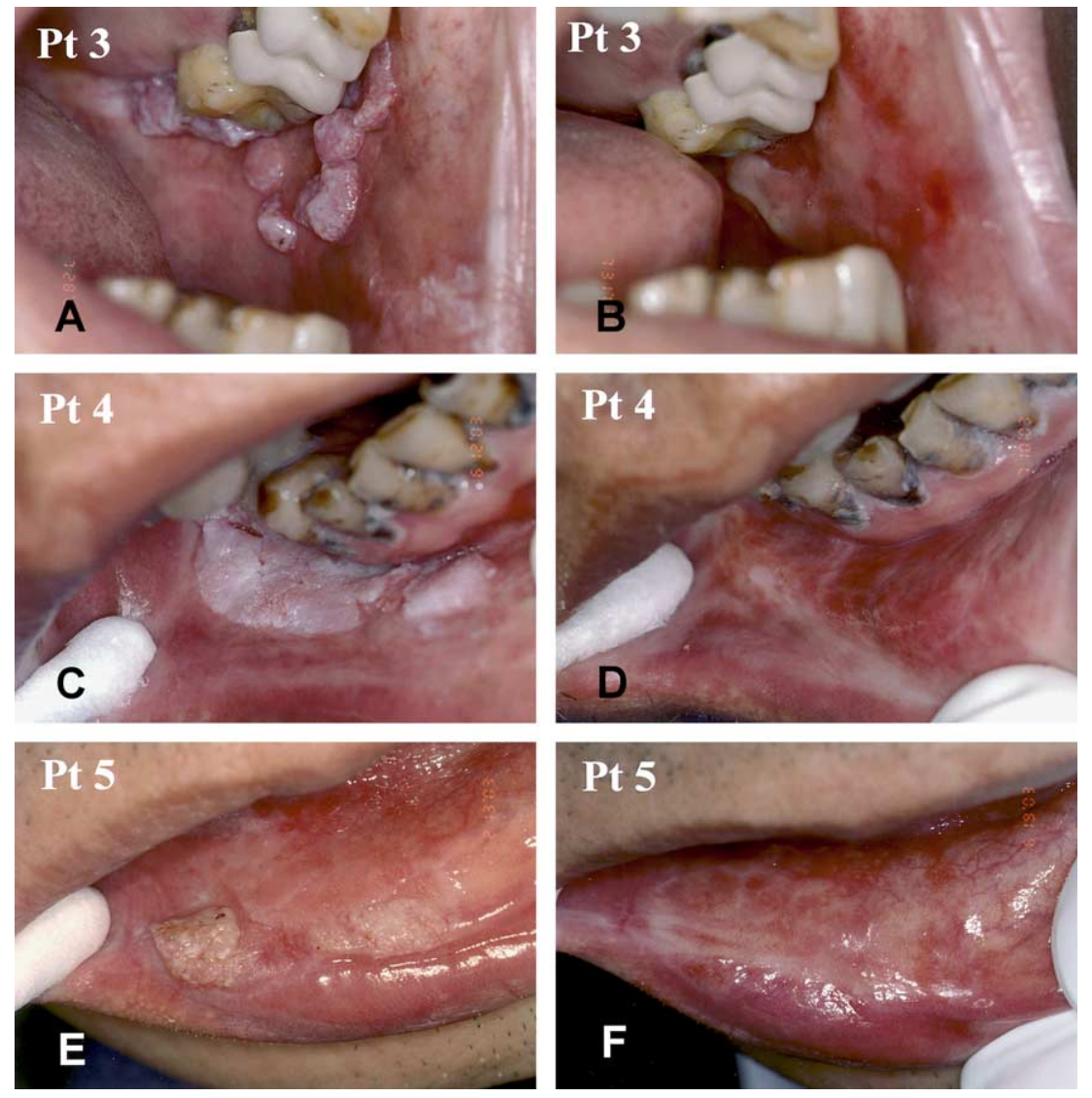

Figure 3 Clinical photographs of patient 3, patient 4 and patient 5 with oral verrucous hyperplasia $(\mathrm{OVH})$. (A) Multiple OVH lesions at the left buccal mucosa of patient 3 before PDT. (B) Clinical picture of patient 3 after 2 treatments of ALA-PDT showing complete regression of the OVH lesions. (C) Two OVH lesions at the right lower labial mucosa of patient 4 before PDT. (D) Clinical picture of patient 4 after 3 treatments of ALA-PDT showing complete regression of the $\mathrm{OVH}$ lesions. (E) Two OVH lesions at the right lower labial mucosa of patient 5 before PDT. (F) Clinical picture of patient 5 after 2 treatments of ALA-PDT showing complete regression of the OVH lesions.

ALA-PDT for $\mathrm{OVH}$ lesions may be at least partially attributed to the new ALA preparation, the new LED light device, and the new treatment protocol used in this study.

PDT with systemically or topically administered ALA has been used for the treatment of oral precancerous and cancerous lesions with good clinical outcomes. ${ }^{47}$ Grant et al. ${ }^{4}$ first used PDT with orally administered ALA to treat four patients with advanced oral SCC. Tumor necrosis was found in three of four patients after PDT. Fan et al. ${ }^{5}$ studied the efficacy of PDT with systemic ALA on 18 oral premalignant and malignant lesions. They found that the depth of tissue necrosis induced by ALAPDT varied from 0.1 to $1.3 \mathrm{~mm}$, but complete epithelial necrosis was present in all cases. All 12 patients with dysplasia showed regression of the lesion to normal or less dysplastic. Some benefit was observed in five of six patients with oral SCC after PDT, but only two became tumor-free. PDT with topical ALA can also be used for treating oral leukoplakia. ${ }^{6,7}$ Kubler et al. ${ }^{6}$ treated 12 oral leukoplakia lesions with PDT after local application of $20 \%$ ALA cream. They observed complete or partial regression in 9 oral leukoplakia lesions 3 months later. Sieron et al. ${ }^{7}$ treated five oral leukoplakia lesions with PDT after topical exposure of 10\% ALA ointment. Complete response was achieved in four out of five oral leukoplakia lesions. The results of above-mentioned investigations and that of the present study indicate that PDT with either systemic or topical ALA is an effective treatment modality for oral premalignant lesions like oral leukoplakia and $\mathrm{OVH}$ and at least has some beneficial effects on oral SCCs.

PDT with systemically or topically administered ALA has also been used for the treatment of premalignant and malignant lesions on the mucosa other than oral mucosa as well as on the skin. Barr et al. ${ }^{8}$ and Gossner et al. ${ }^{9}$ used PDT with systemic 
ALA to treat 15 patients with high-grade dysplasia and 22 patients with mucosal cancer in Barrett's esophagus. They found that high-grade dysplasia was eradicated in all patients and mucosal cancer was eliminated in 17 of $22(77 \%)$ patients. All tumors less than or equal to $2 \mathrm{~mm}$ in thickness were completely ablated. Ackroyd et al. ${ }^{10}$ did a prospective, randomized, double-blind, placebo-controlled trial of PDT with systemic ALA for 36 patients with dysplastic Barrett's esophagus. Of 18 patients in the ALA group, a response was seen in $16(89 \%)$ patients and no dysplasia was seen in esophageal epithelium of patients after PDT. In the placebo group, a decrease in area of $10 \%$ was observed in 2 patients with no change in 16 patients and persistent low-grade dysplasia was found in 12 patients after PDT. Bodner et al. ${ }^{11}$ used PDT with topical ALA to treat $11 \mathrm{HPV}$-positive patients with cervical intraepithelial neoplasia II. They showed that HPV was eradicated in $73 \%$ of the lesions 3 months later and $91 \%$ of the patients were diseasefree after 12-month follow-up. Berger et al.12 performed PDT with topical ALA for 31 patients with recurrent superficial bladder cancer. They found that 16 patients were free of tumor recurrence at an average follow-up of 23.7 months and 15 patients had developed tumor recurrence after a mean of 8.3 months. Waidelich et al. ${ }^{13}$ performed PDT after oral administration of ALA in 24 patients with refractory superficial bladder cancer or carcinoma in situ (CIS). They found that three of the five patients with CIS and 4 of the 19 with papillary cancers were free of recurrence after 12-51 months of follow-up. Three patients were rendered disease-free by repeat ALA-PDT. Topical ALA-PDT has also been used to treat skin lesions like actinic keratosis, Bowen's disease, and basal cell carcinoma with satisfactory results. ${ }^{14,15}$ All of these findings indicate that topical or systemic ALA-PDT is a well-tolerated, tissue-sparing, and effective alternative treatment for premalignant and superficial, less-invasive malignant lesions of the mucosa and skin.

This study used a new formulation of topical ALA preparation that had the following advantages. The liquid form of ALA solution at room temperature became a gel form at body temperature upon contacting the lesional oral mucosa due to a thermoresponsive sol-gel transition of the vehicle. The gel form of ALA preparation was adhesive to the oral mucosa and partially resistant to the dilution of the saliva. In addition, precaution procedures like placing cotton rolls onto the orifices of excretory ducts of major salivary glands and intermittent suctions during the initial 10 min after topical application of ALA were taken for each patient. We believe that these procedures also prevent the dilution of ALA by the saliva and lead to uninterrupted and successful absorption of ALA by the lesional cells.

To the best of our knowledge, most of the previous PDT studies used laser light to treat a variety of premalignant and malignant human lesions; we first tested the efficacy of an LED light irradiation on oral premalignant lesions like $\mathrm{OVH}$. Laser machine can provide light with specific mono-wavelength; however, it is relatively complicated, bulky, heavy, expensive, and unconvenient. The LED light delivery system was simpler, smaller, lighter, cheaper, and more portable than the laser machine. Although an LED light device can only provide light with a range of wavelengths, the result of this topical ALA-PDT study suggests that it is also very effective for treating oral premalignant lesion like $\mathrm{OVH}$. A large-scale experiment is now carrying out in our laboratory to test the clinical efficacy of the LED light irradiation with topical ALA application on oral leukoplakia, erythroplakia, verrucous carcinoma, and superficial SCC.

The majority of previous PDT studies used continuous irradiation for treating a variety of human lesions. ${ }^{4-15}$ In this study, we divided the 1000-s light treatment course into five periods of $180 \mathrm{~s}$ and a period of $100 \mathrm{~s}$. These six periods of light treatment were interrupted by five periods of 3-min rest. Because an efficient PDT needs sufficient and continuous supply of new PpIX and oxygen, multiple 3-min stops were supposed to give the opportunities for tissues to regenerate new PpIX and to obtain new oxygen. It is not clear whether the multi-stop PDT regimen is superior to the non-stop treatment protocol. Further studies are needed to verify the real efficacy of this new multi-stop PDT treatment protocol on human premalignant and malignant lesions.

In summary, this study tested the clinical efficacy of a new treatment protocol of topical ALAPDT for OVH lesions. Complete regression was achieved after 1-3 treatments of ALA-PDT for all lesions. We conclude that PDT using a topical application of $20 \%$ ALA followed by multiple 3-min fractionated irradiations with an LED red light is an effective and successful treatment modality for $\mathrm{OVH}$.

\section{Acknowledgements}

This study was supported by research grants of NSC90-2736-L-002-002 and NSC90-2736-L-002003 from the National Science Council, Taipei, Taiwan. 


\section{References}

1. Dolmans DE, Fukumura D, Jain RK. Photodynamic therapy for cancer. Nat Rev Cancer 2003;3(5):380-7.

2. Kennedy JC, Marcus SL, Pottier RH. Photodynamic therapy (PDT) and photodiagnosis (PD) using endogenous photosensitization induced by 5 -aminolevulinic acid (ALA): mechanisms and clinical results. J Clin Laser Med Surg 1996;14(5): 289-304.

3. Kennedy JC, Pottier RH, Pross DC. Photodynamic therapy with endogeneous protoporphyrin IX: basic principles and present clinical experience. J Photochem Photobiol B: Biol 1990;6(1-2):143-8.

4. Grant WE, Hopper C, MacRobert AJ, Speight PM, Bown SG. Photodynamic therapy of oral cancer: photosensitisation with systemic aminolaevulinic acid. Lancet 1993;342(7): 147-8.

5. Fan KF, Hopper C, Speight PM, Buonaccorsi G, MacRobert AJ, Bown SG. Photodynamic therapy using 5-aminolevulinic acid for premalignant and malignant lesions of the oral cavity. Cancer 1996;78(7):1374-83.

6. Kubler A, Haase T, Rheinwald M, Barth T, Muhling J. Treatment of oral leukoplakia by topical application of 5-aminolevulinic acid. Int J Oral Maxillofac Surg 1998; 27(6): 466-9.

7. Sieron A, Namyslowski G, Misiolek M, Adamek M, KawczykKrupka A. Photodynamic therapy of premalignant lesions and local recurrence of laryngeal and hypopharyngeal cancers. Eur Arch Oto-Rhino-Laryngol 2001;258(7):34952.

8. Barr H, Shepherd NA, Dix A, Roberts DJ, Tan WC, Krasner N. Eradication of high-grade dysplasia in columnar-lined (Barrett's) oesophagus by photodynamic therapy with endogenously generated protoporphyrin IX. Lancet 1996; 348(9027):584-5.

9. Gossner L, Stolte M, Sroka R, Rick K, May A, Hahn EG, et al. Photodynamic ablation of high-grade dysplasia and early cancer in Barrett's esophagus by means of 5-aminolevulinic acid. Gastroenterologia 1998;114(3):448-55.

10. Ackroyd R, Brown NJ, Davis MF, Stephenson TJ, Marcus SL, Stoddard CJ, et al. Photodynamic therapy for dysplastic Barrett's oesophagus: a prospective, double blind, randomized, placebo controlled trial. Gut 2000;47(5):612-7.
11. Bodner K, Bodner-Adler B, Wierrani F, Kubin A, Szolts-Szolts $J$, Spangler B, et al. Cold-knife conization versus photodynamic therapy with topical 5-aminolevulinic acid (5-ALA) in cervical intraepithelial neoplasia (CIN) II with associated human papillomavirus infection: a comparison of preliminary results. Anticancer Res 2003;23(2C):1785-8.

12. Berger AP, Steiner H, Stenzl A, Akkad T, Bartsch G, Holtl L. Photodynamic therapy with intravesical instillation of 5aminolevulinic acid for patients with recurrent superficial bladder cancer: a single-center study. Urology 2003;61(2): 338-41.

13. Waidelich R, Stepp H, Baumgartner R, Weninger E, Hofstetter A, Kriegmair M. Clinical experience with 5-aminolevulinic acid and photodynamic therapy for refractory superficial bladder cancer. J Urology 2001;165(6 Pt 1): 1904-7.

14. Dijkstra AT, Majoie IM, van Dongen JW, van Weelden $H$, van Vloten WA. Photodynamic therapy with violet light and topical 6-aminolaevulinic acid in the treatment of actinic keratosis, Bowen's disease and basal cell carcinoma. J Eur Acad Dermatol Venereol 2001;15(6):550-4.

15. Morton CA, Whitehurst C, McColl JH, Moore JV, Mackie RM. Photodynamic therapy for large or multiple patches of Bowen's disease and basal cell carcinoma. Arch Dermatol 2001;137(3):319-24.

16. Peng Q, Warloe T, Berg K, Moan J, Kongshaug M, Giercksky $\mathrm{KE}$, et al. 5-Aminolevulinic acid-based photodynamic therapy. Cancer 1997;79(12):2282-308.

17. Leunig A, Rick K, Stepp H, Gutmann R, Alwin G, Baumgartner $R$, et al. Fluorescence imaging and spectroscopy of 5-aminolevulinic acid induced protoporphyrin IX for the detection of neoplastic lesions in the oral cavity. Am J Surg 1996;172(12):674-7.

18. Leunig A, Betz CS, Mehlmann $M$, Stepp $H$, Arbogast $S$, Grevers $G$, et al. Detection of squamous cell carcinoma of the oral cavity by imaging 5 -aminolevulinic acid-induced protoporphyrin IX fluorescence. Laryngoscope 2000;110(1): 78-83.

19. Leunig A, Mehlmann M, Betz C, Stepp H, Arbogast S, Grevers $\mathrm{G}$, et al. Fluorescence staining of oral cancer using a topical application of 5-aminolevulininc acid: fluorescence microscopic studies. J Photochem Photobiol B: Biol 2001;60(1): 44-9.

Available online at www.sciencedirect.com 